\title{
Chromosomes in Interphase Nuclei
}

\author{
Riojun Kinosita and Susumu Ohno \\ Department of Cytology, Division of Research, City of Hope Medical Center, Duarte, \\ California, and Departments of Infectious Diseases and Pathology, School of \\ Medicine, University of California at Los Angeles, California, U.S.A.
}

Received November 17, 1955

Chromosomes are not usually recognizable as such during the intermitotic phase (Hughes 1952). It has been assumed, nevertheless, that chromosomes persist in interphase nuclei on the basis of indirect evidence of genetic continuity of chromosomes. Ris and Mirsky (1949) described chromosome-like threads isolated by disintegrating nuclei. Lamb (1949) viewed them as products of the whole nucleus drawn out into elongated bodies which were not equivalent to chromosomes. The procedure utilized may be criticized for inducing modifications of the natural states.

Fell and Hughes (1949), on examining mouse cells in tissue culture, recognized two forms of intranuclear elements, namely chromocenters and chromonemata. The former, granules in form, were visible in the living nucleus by phase-contrast microscopy and Feulgen-positive after fixation, while the latter, fine threads in form, were faintly Feulgen-positive. It has been postulated that the chromocenters are small segments of chromosomes persisting through interphase and that the chromonemata are "backbone" threads of chromosomes from which most Feulgen-positive substances were lost during nuclear reconstruction (Hughes 1952).

Chromatin substances in the nucleus are known to be fine is some cells and coarse in others. According to Serra (1947) the cells in which the chromonemata were readily observed were secretory cells and those in tissues with frequent mitosis. Change occurring in chromosomes during nuclear reconstruction appear to vary according to types of cells. During recent investigations of rat myeloid cells at different stages of maturation (Kinoshita and Ohno 1954, 1955), it was noted that, the more mature the cells were, the more frequently the chromocenters were observed. In other words, a larger number of chromosomal segments appeared to have persisted without losing their staining characteristics. It was also found that the myelocytes, as they advanced in maturation, were hypoploid (Kinoshita and Ohno 1954) and, instead of repeating reproductive mitosis, differentiated to form various types of adult granulocytes by forming such chromosome groupings as characterize the respective nuclear types (Ohno, Kinoshita and Ward 1954).

On the basis of the above considerations, morphological investigations were undertaken on chromosomal elements visualized by an improved technique in interphase nuclei of myeloid cells at various stages of maturation. The 

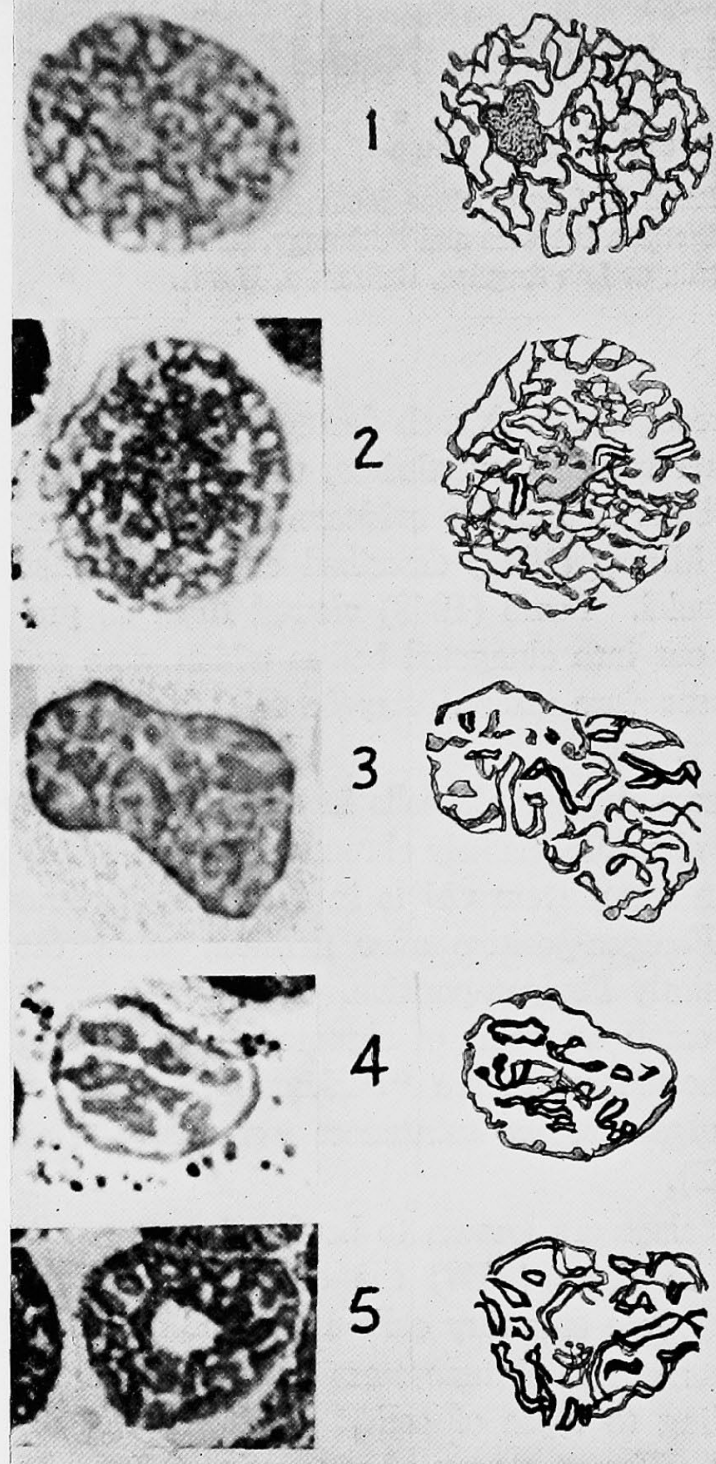

Fig. 1. Photomicrographs of the interphase nuclei of the rat myeloid cells at various stages of maturation (Giemsa; Periplan $15 \times$ and $2 \mathrm{~mm}$. Apo Oel) and their corresponding sketches. Two morphologically distinct chromosomal elements are recognized in the nuclei, namely fine, long, intertwining threads and short, deeply stained rods. The rods demonstrate double-stranded structures. In the myeloblast (1) exclusively the threads forming a network are seen. In the promyelocyte (2) the rods although few in number are present. In the neutrophil mature myelocyte (3) and the basophil mature myelocyte (4) the rods are the predominant elements. The neutrophil leukocyte (5) shows only the rods. findings were compared with telophasic chromosomes of the corresponding types of cells with the object of identifying the elements.

\section{Experiments}

Young, healthy Waynepink-eye rats, weighing about 100 gms., were used for the experiment. Small pieces of femoral bone marrow were surgically removed and rinsed in an equal volume of horse serum. The resulting fluid, in which the myeloid cells were freely suspended, was diluted with an equal volume of 0.05 percent solution of sodium acetate, aspirated in a blood-cell count pipette and incubated at $3^{\circ} \mathrm{C}$ for 20 minutes. A drop of the fluid was spread on a clean microscope slide to make a thin film preparation. This was dried quickly, immediately fixed with one percent solution of chromic acid and then with methanol and stained with Giemsa stain using McJunkin's buffer solution. The cells and nuclei became swollen in the suspending fluid and were flattened as the preparation dried up, so that the fine, intranuclear formal structures were dispersed and made distinctly observable. Types of the cells were determined and their locations were marked. Then the preparations were treated with $1 \mathrm{~N}$ solution of $\mathrm{HCl}$ for 15 minutes at $60^{\circ} \mathrm{C}$, washed with distilled water, immersed in a saturated solution 
of picric acid for 10 minutes, and stained again with Giemsa stain. Some duplicate preparations were stained with Feulgen instead of Giemsa stain. Both the azure dye and the Feulgen stain, known to stain chromosomal substances, demonstrated identical structures, but the Giemsa method was superior in presenting a sharp contour of the fine structures.

In the nuclei two morphologically distinct chromosomal elements were recognized: namely, fine, long, intertwining threads and short, deeply stained rods (Fig. 1). The rods especially showed double-stranded structures clearly. In more mature cells, the chromosomal elements, as a whole, were fewer in number. They were readily countable in the adult granulocytes. It was observed in the previous studies that the mitotic chromosomes were definitely fewer in more mature myeloid cells (Kinoshita and Ohno 1954).

The proportion of the two forms of chromosomal elements varied according to the degree of cell maturation. The myeloblast demonstrated exclusively the intertwining, fine threads forming a network (Fig. 1-1). The promyelocyte presented a small number of the rods in addition to the network of the threads (Fig. 1-2). Either of these cells, especially the former, are to be concerned with reproductive mitosis (Ohno, Kinoshita and Ward 1954) or at the intermitotic phase of the interphase-mitosis cycle (Fig. 2). In the mature myelocyte, the fine threads were scanty and no longer made up a network, while the rods became the predominant elements (Figs. 1-3 and 1-4). The mature myelocyte is capable of entering into mitosis, in most

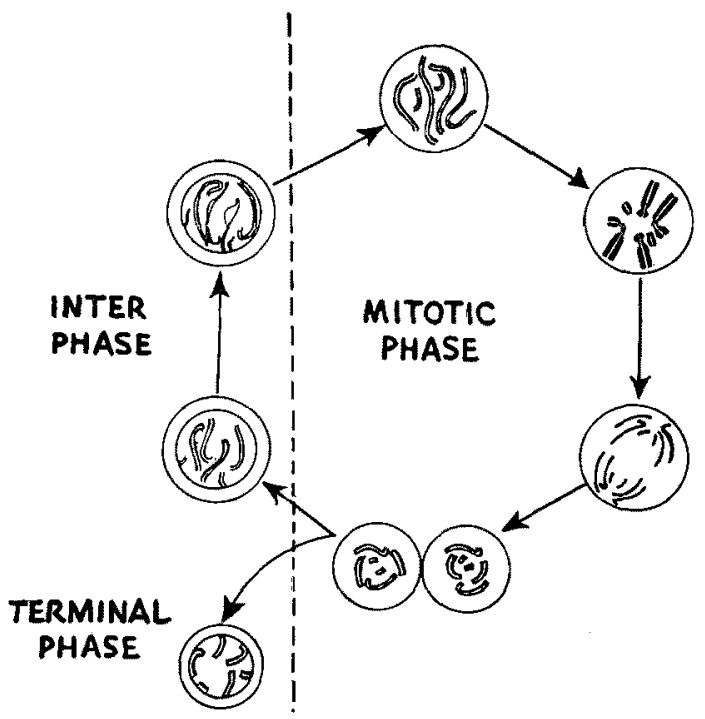

Fig. 2. A diagram of the interphase-mitosis cycle and the terminal mitotic phase followed by the terminal resting phase.

cases producing daughter cells in forms of adult granulocytes (Ohno, Kinoshita and Ward 1954). The mitosis in this case is a terminal one associated with differentiation (Fig. 2). On the other hand, the adult granulocyte nucleus contained the rods only (Fig. 1--5). The adult granulocyte is at the terminal, resting stage and no longer able to undergo mitosis (Kinoshita and Ohno 1955).

Comparative observations, employing the same preparation as used in the above observations, were undertaken on the mitotic chromosomes at the stage of nuclear reconstruction at late telophase to determine the interrelationships between these and the structural elements demonstrated in the interphase nucleus. The chromosomes of the myeloblast at this stage of mitosis became 
stretched to long, fine threads which resembled the interwining, fine threads in the interphase nucleus of the myeloblast. In some myelocytes the telophasic chromosomes, for the most part, showed similar change, while a few others remained short, rod-shaped, structurally double-stranded, and deeply stained, on the whole presenting an appearance resembling that of the chromosomal elements demonstrated in the promyelocyte nucleus. These cells were undergoing reproductive mitosis. On the other hand, the mature myelocyte formed telophasic daughter cells with fewer chromosomes most of which consisted of the rod type like the chromosomal elements in the adult granulocyte nucleus. The similarities thus demonstrated between the telophasic chromosomes and the intranuclear chromosomal elements suggested that the latter were the continuant elements of the mitotic chromosomes.

\section{Summary}

Azure or Feulgen positive, well-defined elements were demonstrated in the interphase nuclei of rat myeloid cells at various stages of maturation.

The elements were fewer in number in more mature cells. They included two forms: namely long, fine threads and short, double-stranded, deeply stained rods. The proportion of these components varied according to maturation of the cell. The threads occurred in the myeloblasts and the promyelocytes exclusively or predominantly, while the adult granulocytes contained only rods. The former group of cells are capable of mitosis and the latter are not. The elements were similar in form and stainability to the mitotic chromosomes at the stage of nuclear reconstruction. The latter also included two forms which proportion varied according to maturation of the cell being formed, similarly as observed in the interphase cells. The similarities thus shown suggested that the chromosomal elements demonstrated in the interphase nuclei are continuants of the mitotic chromosomes.

\section{References}

Fell, H. B., and Hughes, A. F. 1949. Quart. J. Microsc. Soc. 90; 355.

Hughes, A. 1952. Mitotic Cycle, p, 30. Butterworks Scientific Publications, London.

Kinoshita, R., and Ohno, S. 1954. Naturwiss. 41: 381.

Kinoshita, R., and Ohno, S. 1955, Proc. Amer. Assoc. Cancer Res. 1: 28.

Lamb, W. G. P. 1949. Nature, London 164: 109.

Ohno, S., Kinoshita, R., and Ward, J. P. 1954. Naturwiss, 41 (12): 288.

Ris, H., and Mirsky, A. E. 1949. J. Gen. Physiol. 32: 489.

Serra, J. A. 1947. Symp. Quant. Biol. 12: 192. 